\title{
Understanding of Dry Eye Syndrome in Traditional Chinese Medicine
}

\author{
Jingxiang Chen ${ }^{1}$, Jingan Tong2* \\ ${ }^{1}$ Shaanxi University of Traditional Chinese Medicine, Xianyang 712000, China \\ ${ }^{2}$ Affiliated Hospital of Shaanxi University of Traditional Chinese Medicine, Xianyang 712000, China \\ *Corresponding author: Jingan Tong, tja1962@126.com
}

\begin{abstract}
Explore the current status of Chinese medicine understanding and prevention and treatment of dry eye, so as to improve the efficacy of Chinese medicine in the diagnosis and treatment of dry eye.
\end{abstract}

Keywords: Traditional Chinese medicine; Dry eye; Understanding; Review

Publication date: November 2021; Online publication: November 30, 2021

\section{Introduction}

Dry eye is one of the most common chronic ocular surface diseases. At present, due to the popularity of video terminals and the aging of the population, the incidence of dry eye is gradually increasing and tends to be younger. The global incidence of dry eye is 5.5\% 33.7\%, and the incidence in my country is $21 \% \sim 30 \%$ [1].

\section{TCM's understanding of dry eye syndrome}

\subsection{Disease name}

In Chinese ophthalmology, dry eye syndrome belongs to the category of "White Astringency." In "Yuanjiqiwei": "It is neither red nor swollen, not refreshing, sandy and faint, and the name is white and astringent [2]." Wang Kentang of the Ming Dynasty described it as "Dry and Dim" in the book "Zheng Sheng ${ }^{[3]}$." In "Theories of the Sources and Phenomenon of All Diseases," there is the title of "The Eyes are Astringent ${ }^{[4]}$."

\section{Etiology and pathogenesis}

\subsection{Liver work}

The head is the liver orifice, born in the kidney, and used for the heart. If the liver blood is sufficient, the wood qi rises, and the blood flow reaches the eye, the spirit is bright. Excessive appetite, exhaustion of eyesight, etc. can deplete qi and blood, cause insufficient qi and blood in the liver, lack of nourishment of blood or dark consumption of essence, qi and yin fluid, inflammation of deficiency fire, decoction of body fluid, diminished light, and astringent eyes. Fang Yuting et al. ${ }^{[5]}$ believed that excessive eye use broke the human body's circadian rhythm, disrupted the law of the ebb and flow of yin and yang, and led to imbalance between yin and yang and eye disease.

\subsection{Liver and kidney yin deficiency}

"Mujing Dacheng·Volume 2·Eighty-one Symptoms" says: "This visceral manifestation of fire, although the true essence may not be absent, but the self-induced evil and delusional consumption of ointment." It 
is believed that this disease is caused by a lack of fire. It is closely related to liver and kidney yin deficiency. Deficiency of kidney yin, lack of source of liver yin, water does not contain wood, and relatively hyperactive liver yang, causing inflammation of liver fire and damage to eye fluid. The liver and kidney yin fluid are depleted, and the fire is uncontrollable. The phase fire is fierce, and violent movement can torture the true yin, and the lack of true yin can damage the liver yin, so the eyes are astringent. Lian Haihong ${ }^{[6]}$ believes that deficiency of liver and kidney yin is the root, hyperactivity of yang is the mark, the mixture of deficiency and excess, and liver heat are the main causes of modern dry eye disease.

\subsection{Lung yin deficiency}

"Surveying Yaohan • Volume 3 • White Pain" says: "White astringency... is the fire of qi and hidden, damp and hot spleen, lungs and collaterals, which are more common in autumn ${ }^{[7]}$." Those with dryness have inherent yin and yang, their sex is dry and easy to damage body fluids, and those with lungs are exposed to autumn qi, and suffer from dry evils, and damage the lungs first. Lung governs the body's qi, and when there is constant declining, the five internal organs are closed, the eyes and collaterals are unblocked, and the divine light is more. Li Pingshan ${ }^{[8]}$ believes that the dryness of the eyeballs is caused by "dryness" injuring the five internal organs, causing the lungs, liver, and kidneys to injure fluid consumption and loss of normal physiological functions.

\subsection{Deficiency of spleen and weak qi}

"The spleen is the head of the yin, and the eye is the bloodline. Therefore, if the spleen is weak, the essence of the five internal organs is lost and cannot be attributed to the eye ${ }^{[9]}$." The role of the spleen and stomach" $\mathrm{s}$ axis is to grow the four internal organs, metabolize the blood and vitality, and lose it to the eyes. If the function of the spleen and stomach is lost, the energy of the blood and the essence of energy is deprived, and the source of energy is lost.

\section{The method of traditional Chinese medicine to treat dry eye \\ 4.1. Acupuncture treatment}

Wen Yong et al. ${ }^{[10]}$ divided 80 cases into two groups. In the acupuncture group, 40 cases used meridian and viscera syndrome differentiation acupuncture combined with thunder fire moxibustion. $77.5 \%$ and $45.0 \%$ in the drug group. Duan Jianwei ${ }^{[11]}$ divided 62 cases into 2 groups. 32 cases in the experimental group were treated with artificial tears and were treated with magpie-dengmei acupuncture. 30 cases in the control group were treated with artificial tears. The total effective rate was $90.63 \%$ in the experimental group. The control group was $70.00 \%$.

\subsection{Chinese medicine ultrasonic atomization treatment}

Li Jianliang et al. ${ }^{[12]}$ divided 120 cases into an observation group and a control group, each with 60 cases (120 eyes). The observation group was treated with Shuyanfang ultrasonic nebulization combined with local instillation of sodium hyaluronate eye drops, and the control group was treated with sodium hyaluronate Eye drops eye drops treatment. The total effective rate of the observation group was $90 \%$, and that of the control group was $65 \%$. Huang Yufeng et al. ${ }^{[13]}$ divided 60 patients (120 eyes) into Chinese medicine ultrasonic atomization combined with artificial tears group (treatment group) and artificial tears (control group). The total effective rate of the treatment group was higher than that of the control group $(\mathrm{P}<0.05)$.

\subsection{Massage therapy}

Wang $\mathrm{Yu}{ }^{[14]}$ divided 80 cases into experimental group and control group. Both groups were treated with 
sodium hyaluronate eye drops, while the experimental group was treated with eight methods of eye massage combined with five-element music therapy. The total effective rate of treatment in the experiment ${ }^{7}$ al group was higher than that in the control group $(\mathrm{P}<0.05)$. Huang Tingqiu et al. ${ }^{[15]}$ divided 106 cases into a combination group and a control group. The control group was treated with traditional Chinese medicine atomized fumigation, and the combination group was treated with traditional Chinese medicine atomized fumigation combined with humidifying eye massager. The total effective rate of the combined group was $94.23 \%$, and the control group was $78.10 \%$.

\section{Summary}

In the future, we should focus on the advantages of the overall adjustment of traditional Chinese medicine, take into account some parts, use modern medical science and technology, and rationally use multiple methods to comprehensively prevent and treat dry eye, improve the level of traditional Chinese medicine in the prevention and treatment of dry eye, and provide more for the prevention and treatment of dry eye.

\section{Disclosure statement}

The authors declare no conflict of interest.

\section{References}

[1] Li P, Li J, Huang S, et al., 2019, Traditional Chinese Medicine Intervention and Nursing Measures for Patients with Dry Eye. Nursing Research, 33(22): 3984-3987.

[2] Wang Y, 2018, Clinical Study of Yangyin Qingfei Decoction Combined with Sodium Hyaluronate Eye Drops in the Treatment of Lung-Yin Deficiency Dry Eye. Henan University of Traditional Chinese Medicine.

[3] Zeng S, Wang B, 2016, Efficacy of Dextran 70 Eye Drops Combined with Traditional Chinese Medicine in the Treatment of Dry Eye after Cataract Surgery. Guangxi Medicine, 38(04): 577-578.

[4] Chen T, Tao H, Zheng Q, 2017, Analysis of Medical Records of Acupuncture and Moxibustion Treatment of Water-Deficiency Dry Eye. Modern Distance Education of Chinese Medicine, 15(09): 107-108.

[5] Fang Y, Liu Y, 2019, Excessive Correlation Eye and Dry Eye, Liaoning University of Traditional Chinese Medicine, 21(08): 192-195.

[6] Lian H, Yin P, 2016, Efficacy Observation of Hung Yuk's Heat Treatment for Dry Eye, Chinese Medicine, 34(05): 1259-1262.

[7] Li J, 2017, Clinical Observation on Treatment of Dry Eye with Meibomian Gland Dysfunction Based on Syndrome Differentiation. Yunnan College of Traditional Chinese Medicine.

[8] Li P, 2012, Summary of Clinical Research on Dry Eye Syndrome in Traditional Chinese Medicine. Global Chinese Medicine, 5(03): 237-240.

[9] Zhang B, Liu H, 1997, On the Relationship Between the Eye and The Stomach, Hebei Traditional Chinese Medicine, (04): 3-4.

[10] Wen Y, Wu X, Qin Z, 2016, Efficacy Evaluation of Acupuncture and Moxibustion on the Treatment of Dry Eye Syndrome During Perimenopausal Period. Guangxi Medicine, 38(08): 1149-1151.

[11] Duan J, Duan L, 2019, To Explore the Clinical Efficacy of Artificial Tears Combined with Acupuncture in the Treatment of Dry Eye. Digest of World Latest Medical Information, 19(53): 187-188.

[12] Li J, Wang R, Du H, et al., 2015, Shuyanfang Ultrasonic Nebulization Combined with Sodium Hyaluronate Eye Drops in the Treatment of 60 Cases of Dry Eye. Jiangxi Traditional Chinese Medicine, 46(09): 58-59. 
[13] Huang Y, Wang F, et al., 2016, Clinical Observation on the Treatment of Dry Eye with Chinese Medicine Ultrasonic Atomization Smoked Eyes Combined with Artificial Tears. Journal of Qiannan Medical College, 29(01): 30-32+70.

[14] Wang Y, 2019, Efficacy of the Eight Methods of Eye Massage Combined with the Five-Element Music Therapy in the Auxiliary Treatment of Dry Eye. Clinical Medical Research and Practice, 4(11): 97-99.

[15] Huang T, Mo J, Chen H, et al., 2016, Observation on the Improvement of Symptoms of Dry Eye Patients with Humidifying Eye Massager Combined with Traditional Chinese Medicine Atomization Fumigation. Zhejiang Journal of Traditional Chinese Medicine, 51(12): 865-866. 